\title{
Quantification of anterior chamber reaction after intravitreal injections of conbercept and ranibizumab: a pilot study
}

\author{
Xulong Liao ${ }^{1}$ Chuang Jin ${ }^{1}$ - Weiqi Chen $\mathbb{1}^{1} \cdot$ Guihua Zhang ${ }^{1} \cdot$ Ling-Ping Cen ${ }^{1}$ Danny Siu-Chun $\mathrm{Ng}^{2}$. \\ Haoyu Chen $\mathbb{D}^{1}$
}

Received: 4 March 2019 / Revised: 13 June 2019 / Accepted: 16 June 2019 / Published online: 18 July 2019

(c) The Royal College of Ophthalmologists 2019

Intraocular inflammation is an important adverse reaction after intravitreal injection of anti-vascular endothelial growth factor (VEGF) agents. The incidence of noninfectious inflammation after anti-VEGF therapy was reported ranging from 0.02 to $0.55 \%[1,2]$. However, some milder intraocular inflammations could have been missed in busy clinics and underreported. Anterior segment optical coherence tomography (AS-OCT) has been used as a sensitive and reproducible measurement to visualise and quantify inflammatory cells in the anterior chamber [3]. In this retrospective study, we used AS-OCT to investigate the incidence and risk factors of anterior chamber reaction after intravitreal injections of anti-VEGF agents.

This study was approved by the Institutional Review Board of the Joint Shantou International Eye Center, Shantou University and the Chinese University of Hong Kong and in agreement with the tenets of the Declaration of Helsinki. Informed consent was obtained from all participants at enrolment. Totally 128 eyes were included in this study. Swept-source AS-OCT (CASIA SS-1000, Tomey, Nagoya, Japan) was used to detect aqueous cells before injection and 1 day after injection. The cells were counted by two masked and independent observers manually. The number of cells was determined by taking the average of the vertical and the horizontal high definition scan images.

There were 39 cases $(30.5 \%)$ with increased $\geq 1$ cell in anterior chamber, which was defined as positive reaction

These authors contributed equally: Xulong Liao, Chuang Jin

Haoyu Chen

drchenhaoyu@gmail.com

1 Joint Shantou International Eye Center, Shantou University \& the Chinese University of Hong Kong, Shantou, China

2 Department of Ophthalmology \& Visual Sciences, the Chinese University of Hong Kong, Hong Kong, China
(Fig. 1). Of them, 13 cases $(10.2 \%)$ had increased $\geq 10$ cells in anterior chamber. There was no significant difference between positive and negative groups in age, gender, diagnosis or history of injection. Patients who received conbercept had higher incidence of positive anterior chamber reaction compared with those who received ranibizumab ( 44.7 vs. $24.5 \%, p=0.02, \chi^{2}$ test, Odds ratio $=$ 2.502, 95\% confidence interval: 1.124-5.569) (Table 1). The incidence of increased $\geq 10$ cells was also higher in the patients received conbercept compared to ranibizumab (13.2 vs. $8.8 \%, \mathrm{OR}=1.553)$, although the difference was not statistically significant ( $p=0.465, \chi^{2}$ test). There was no statistical discrepancy between positive and negative group in intraocular pressure and best-corrected visual acuity changes at 7 days and central macular thickness changes at 1 day. None of the patients complained symptom of floating or vision decrease. None of the patients needed treatment for anterior chamber cells. In 18 patients of positive group had re-examinations of AS-OCT 7 days after injection. The cells in the anterior chamber had disappeared completely in 16 patients and decreased to $\leq 2$ cells in the other two patients.

The incidence of anterior chamber reaction in our study $(30.5 \%)$ is much higher compared to the noninfectious inflammation reported in the literature (ranged from 0.02 to $0.55 \%)[1,2]$. It is because of the prospective design of current study and the use of AS-OCT which had higher sensitivity in detecting anterior chamber cells comparing to slit-lamp biomicroscope.

Our study found that the incidence of anterior chamber reaction was significantly more common in conbercept than ranibizumab. This result is consistent with literature reports that aflibercept have higher incidence of noninfectious ocular inflammation than ranibizumab [1, 4]. Both conbercept and aflibercept are fusion proteins that composed of human immunoglobulin Fc region and VEGF receptor key domains, while ranibizumab is the Fab fragment of immunoglobulin and there is no $\mathrm{Fc}$ region. The $\mathrm{Fc}$ region on 
Fig. 1 Anterior segment optical coherence tomography images of cases with negative and positive anterior chamber reaction after intravitreal injection of anti-VEGF agents

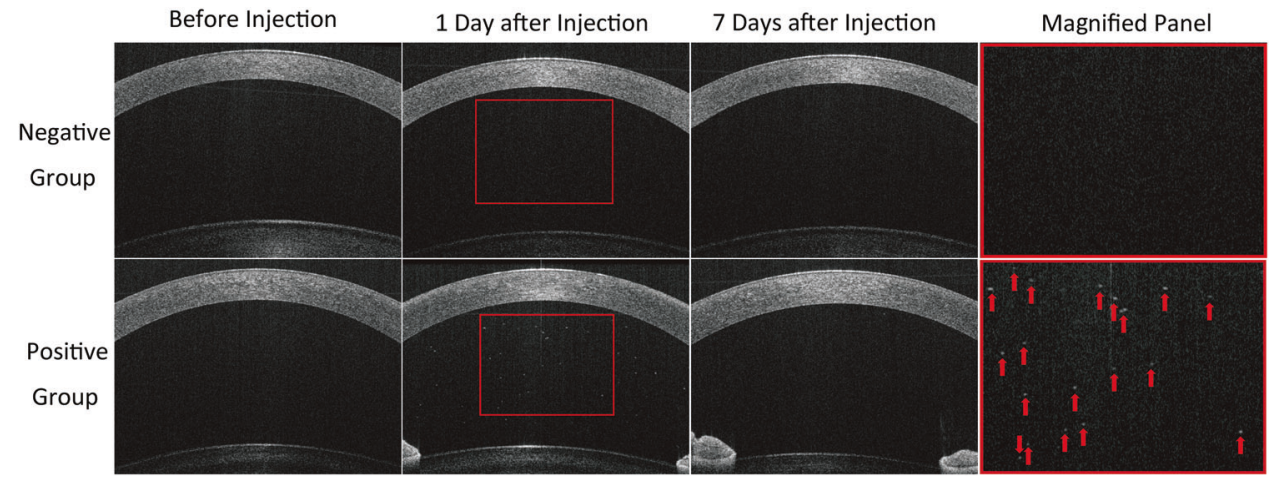

Table 1 Comparison of the characters between the groups with positive and negative anterior chamber reaction after anti-VEGF therapy

\begin{tabular}{llll}
\hline & Positive & Negative & $P$ value \\
\hline$N$ & 39 & 89 & \\
Age (years) & $58.00 \pm 13.13$ & $57.37 \pm 10.19$ & $0.147^{*}$ \\
Gender: Male & $20(27.8 \%)$ & $52(72.3 \%)$ & $0.453^{\#}$ \\
Female & $19(33.9 \%)$ & $37(41.6 \%)$ & \\
Diagnosis: & $24(36.4 \%)$ & $42(63.6 \%)$ & $0.135^{\#}$ \\
Choroidal diseases & & & \\
Retinal diseases & $15(24.2 \%)$ & $47(75.8 \%)$ & \\
History of & $17(32.7 \%)$ & $35(67.3 \%)$ & $0.651^{\#}$ \\
injection: Yes & & & \\
No & $22(28.9 \%)$ & $54(71.1 \%)$ & $0.023^{\#}$ \\
Drug: Conbercept & $17(44.7 \%)$ & $22(55.3 \%)$ & \\
Ranibizumab & $21(24.4 \%)$ & $68(75.6 \%)$ & \\
\hline
\end{tabular}

*independent $t$-test; ${ }^{\#} \chi^{2}$ test

conbercept or aflibercept may activate the Fc receptor on immune cells and receptor mediated cytotoxicity [5].

In conclusion, our study revealed significant proportion of cases developed increased anterior chamber reaction after intravitreal injections of anti-VEGF agents. Conbercept had higher risk of anterior chamber reaction compared to ranibizumab.

\section{Compliance with ethical standards}

Conflict of interest The authors declare that they have no conflict of interest.

Publisher's note: Springer Nature remains neutral with regard to jurisdictional claims in published maps and institutional affiliations.

\section{References}

1. Williams PD, Chong D, Fuller T, Callanan D. Noninfectious vitritis after intravitreal injection of anti-VEGF agents: variations in rates and presentation by medication. Retina. 2016;36:909-13.

2. Kim JY, You YS, Kwon OW, Kim SH. Sterile inflammation after intravitreal injection of aflibercept in a Korean population. Korean J Ophthalmol. 2015;29:325-30.

3. Invernizzi A, Marchi S, Aldigeri R, Mastrofilippo V, Viscogliosi F, Soldani A et al. Objective quantification of anterior chamber inflammation: measuring cells and flare by anterior segment optical coherence tomography. Ophthalmology. 2017;124:1670-7.

4. Souied EH, Dugel PU, Ferreira A, Hashmonay R, Lu J, Kelly SP. Severe ocular inflammation following ranibizumab or aflibercept injections for age-related macular degeneration: a retrospective claims database analysis. Ophthalmic Epidemiol. 2016;23:71-9.

5. Quast I, Keller CW, Maurer MA, Giddens JP, Tackenberg B, Wang LX, et al. Sialylation of $\mathrm{IgG} F \mathrm{Fc}$ domain impairs complement-dependent cytotoxicity. J Clin Investig. 2015;125: 4160-70. 